\title{
Spinal Muscular Atrophy with Preserved Deep Tendon Reflexes
}

\author{
Jyotindra Narayan Goswami ${ }^{1} \cdot$ Jitendra Kumar Sahu $^{1} \cdot$ Pratibha Singhi $^{1}$
}

Received: 3 August 2017 / Accepted: 12 October 2017 / Published online: 15 November 2017

(C) Dr. K C Chaudhuri Foundation 2017

To the Editor: Spinal muscular atrophy (SMA) is an autosomal recessive neuromuscular disorder in which there is areflexia [1]. We report an atypical case of a child with SMA and preserved deep tendon reflexes.

A 6-y-old boy presented with progressively increasing walking difficulty from five years of age. At presentation, he could not walk briskly, climb upstairs or get up from sitting position unaided. He did not have slippage of slippers, upper limb weakness, tingling/numbness, tremor, ataxia, bladder/bowel symptoms. His anthropometry, vital parameters, skull/spine, higher-mental functions, speech, cranial nerves and tone were normal. He had bilaterally reduced power of hip-flexion and extension (Grade III/V). Power across other joints was normal. He had brisk ankle jerks, ill-sustained clonus and paraspinal fasciculations. Other deep tendon jerks were normally elicitable. Plantars were flexor. There were no tongue-fasciculations or acral-tremors. Examination of sensory, cerebellar, autonomic functions and other systems was unremarkable. His creatine phosphokinase, thyroid function test, spinal MRI and nerve conduction study were normal. Electromyography revealed fasciculations and fibrillations in vastus lateralis and first dorsal interosseus. Hence, gene mutation analysis for Survival Motor Neuron 1 ( $S M N$ 1) was obtained which revealed exon-7 deletion from both copies of SMN 1 gene establishing diagnosis of SMA. Child was started on physiotherapy. Follow-up after six months revealed generalized areflexia with static functional status.

Pratibha Singhi

doctorpratibhasinghi@gmail.com

1 Pediatric Neurology and Neurodevelopment Unit, Department of Pediatrics, Post Graduate Institute of Medical Education \& Research, Chandigarh 160012, India
Muscular weakness, areflexia and fasciculations are hallmarks of SMA [2]. Brisk tendon reflexes posed major diagnostic dilemma in index child. Hence, muscle/ spinal cord pathology were initially excluded. Literature search did not reveal any instance of SMA with preserved reflexes. We hypothesize that the child presented when partial anterior horn cell function was present. Later, he developed areflexia with progressive anterior horn cell degeneration.

Our case attempts to sensitize pediatricians about the rare possibility of preserved reflexes in SMA early in its temporal course. Clinical follow-up, electrophysiology and genetic testing help in this scenario.

\section{Compliance with Ethical Standards}

Conflict of Interest None.

Source of Funding None.

\section{References}

1. Kolb SJ, Kissel JT. Spinal muscular atrophy: a timely review. Arch Neurol. 2011;68:979-84.

2. Wang CH, Finkel RS, Bertini ES, et al. Consensus statement for standard of care in spinal muscular atrophy. J Child Neurol. 2007;22:1027-49. 